\title{
Adipositas im Kindesalter und Asthma bronchiale
}

\section{Zwischen Adipositas- und Asthma- prävalenz besteht eine Assoziation. Patienten mit Asthma bronchiale und Adipositas sprechen schlechter auf Glukokortikoide an und sind thera- peutisch schlechter zu kontrollieren als normalgewichtige Patienten. Die Ursachen hierfür sind noch unklar.}

- Die Adipositas und das Asthma bronchiale stellen sowohl im Erwachsenenals auch im Kindesalter eine große gesundheitspolitische Herausforderung dar, da ihre Prävalenz in den letzten 20 Jahren in allen westlichen Industrieländern sukzessive zugenommen hat. Das männliche Geschlecht ist sowohl von der Adipositas als auch vom Asthma bronchiale häufiger betroffen als das weibliche Geschlecht.

Da zum Einfluss des Körpergewichts auf die Prävalenz des Asthma bronchiale im Kindesalter bisher noch keine Daten über Geschlechts- und Rassenunterschiede vorliegen, sollte diese Fragestellung anhand der Daten aus zwei großen Asthmakohortenstudien, die insgesamt 2791 Kinder und Jugendliche mit einem mittleren Alter von 12,8 Jahren umfasste, untersucht werden. Im Vergleich $\mathrm{zu}$ den normgewichtigen alters- und geschlechtsgleichen Kontrollgruppen ergab sich bei den männlichen Asthmapatienten eine um 30\% höhere Prävalenz für eine Adipositas. Im Gegensatz

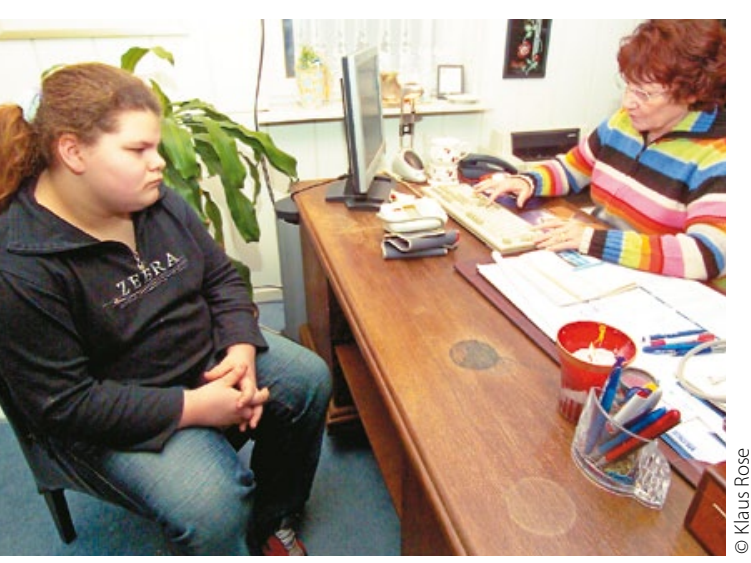

Bei adipösen Kindern ist Asthma schwer kontrollierbar. zu den Mädchen hatten die Knaben ein schlechter kontrollierbares Asthma. Sie benötigten mehr Antiasthmatika, sprachen schlechter auf die Therapie an und hatten permanent eine schlechtere Lungenfunktion und mehr Symptome.

Im Unterschied zu den Knaben zeigten die übergewichtigen Mädchen eine Abhängigkeit ihres Asthmaschweregrades von der ethnischen $\mathrm{Zu}$ gehörigkeit. Übergewichtige bzw. adipöse schwarze Mädchen hatten gegenüber normgewichtigen schwarzen Mädchen mit Asthma bronchiale ein besser einstellbares und therapierbares Asthma (Odds Ratio 0.65 vs. 1 für die Adipösen) als mexikanische Mädchen gegenüber ihrer normgewichtigen Vergleichsgruppe (1,91 vs. 1 für die Adipösen)

- L.N. Borell et al.

Childhood obesity and asthma control in the

GALA II and SAGE II studies. Am J Respir Crit

Care Med 2013; 187: 697-702

\section{Kommentar}

Zwei große Kohortenstudien zeigten, dass zwischen Asthma und Adipositasprävalenz bei männlichen Patienten eine Assoziation besteht. Neu ist, dass weibliche übergewichtige Asthmapatienten eine Abhängigkeit der Kontrollierbarkeit ihres Asthma bronchiale von der ethnischen Zugehörigkeit aufweisen. Schwarze adipöse Patienten etwa sprechen besser an als mexikanischstämmige Patienten im Vergleich zu ihren normgewichtigen Kontrollen mit der gleichen ethnischen Zugehörigkeit. Da in Deutschland nicht nur die die Zahl der adipösen Kinder, sondern auch die der Migranten mit unterschiedlichem ethnischen Hintergrund stetig zunimmt, sollte bei einer unterschiedlichen individuellen Kontrollierbarkeit des Asthma bronchiale daran gedacht werden, dass möglicherweise hierfür Körpergewicht, Geschlecht und Migrationshintergrund verantwortlich sein können.

D. REINHARDT =

\section{Statine können Muskeln und Gelenken schaden}

\begin{abstract}
Vor allem körperlich aktive Patienten, die Statine einnehmen, haben mehr Erkrankungen des Bewegungsapparats als vergleichbare Personen, die nicht mit Statinen behandelt werden.
\end{abstract}

- In einer retrospektiven Studie untersuchten Forscher in den USA den Zusammenhang zwischen Statineinnahme und Muskel- und Gelenkbeschwerden. Sie verglichen ca. 7000 Patienten mit und gleich viele ohne Statineinnahme über ca. vier Jahre und fanden unter den Patienten, die Statine einnahmen, rund $20 \%$ mehr Fälle von Muskelschmerzen und -schwäche sowie ca. 10\% mehr Tendopathien und Arthrosen.

\section{Mansi et al.}

Statins and musculoskeletal conditions, arthropathies and injuries. JAMA 2013 (online first, 3. Juni); doi: 10.1001/jamainternmed.2013.6184.

\section{Kommentar}

Diese retrospektive Studie ist ein erneuter Hinweis darauf, dass Statine, wahrscheinlich über ihre hemmende Wirkung auf das Coenzym Q10, nachteilige Wirkungen an Muskel, Sehnen, Knochen und Gelenken ausüben können. Insbesondere bei älteren Menschen, deren muskuläre Kompetenz nicht selten tiefgreifende Konsequenzen auf Gehfähigkeit, Alltagsbewältigung und Selbständigkeit hat, sollte man vorsichtig abwägen. Gut gemeinte kardiovaskuläre Prävention kann so schnell ins Gegenteil umschlagen, weshalb nicht nur das organfokussierte kardiologische, sondern auch das funktionsbezogene geriatrische Votum gehört werden sollte.

J. ZEEH - 